\title{
Mathematical Modeling of Nutrient Release from Capsulated Fertilizers
}

\author{
Viktoriia Vakal' ${ }^{1}$ Ivan Pavlenko ${ }^{2 *}$, Serhii Vakal', Larisa Hurets ${ }^{3}$, Marek Ochowiak ${ }^{4}$ \\ 1 Scientific and Research Institute of Mineral Fertilizers and Pigments, Sumy State University, Kharkivska St., 40003 Sumy, Ukraine \\ 2 Department of General Mechanics and Machine Dynamics, Faculty of Technical Systems and Energy Efficient Technologies, \\ Sumy State University, 2 Rymskogo-Korsakova St., 40007 Sumy, Ukraine \\ ${ }^{3}$ Department of Applied Ecology, Faculty of Technical Systems and Energy Efficient Technologies, Sumy State University, \\ 2 Rymskogo-Korsakova St., 40007 Sumy, Ukraine \\ ${ }^{4}$ Department of Chemical Engineering and Equipment, Faculty of Chemical Technology, Poznan University of Technology, \\ 5 M. Sklodowska-Curie Sq., 60-965 Poznan, Poland \\ * Corresponding author, e-mail: i.pavlenko@omdm.sumdu.edu.ua
}

Received: 29 March 2019, Accepted: 06 June 2019, Published online: 13 September 2019

\begin{abstract}
The article is devoted to the study of reducing the technogenic load from high-dose nitrogen pollution of the soil layer by encapsulating granules of nitrogen fertilizers in slowly soluble phosphate-containing membranes. The process of dissolving and washing out of the primordial soil layer of the nutrients from urea, encapsulated by superphosphate shells in soil columns, was studied according to agrochemical techniques. The purpose of the work is to determine the parameters of the investigated process, based on which the previously developed physical model of soil washing of moving nitrogen forms, based on experimental data. The process of changing the loss of nitrogen from the soil when using different types of fertilizers depending on the amount of washing was studied by simulation. Mathematically, the process is described in general terms as a linear differential equation of the first order in partial derivatives. The solution of the equation under the initial and boundary conditions allowed to calculate the parameters of the model describing the nature of changes in the losses of nitrogen from the soil column. Comparison of calculated and experimental data showed a coincidence of values with a correlation coefficient above 0.96 for investigated nitrogen fertilizers with different composition of the phosphate-containing coating.
\end{abstract}

Keywords

carbamide, capsulated fertilizers, phosphate-containing membrane, regression analysis, dissolution rate

\section{Introduction}

Ecological safety of nitrogen fertilizer granules encapsulated by phosphate-containing coatings when theirs introducing into the soil is due to the necessity of ensuring the needs of agricultural crops by this type of nutrients and requires to prevent the local supersaturation of the soil layer by nitrates and heavy metals by rapid dissolution of chemical elements of fertilizers. In this case, the physical and chemical properties of mineral fertilizers play an extremely important role.

On the one hand, the high solubility of nitrogen-containing fertilizers significantly increases the content of nitrates in soil ecosystems. On the other hand, it leads to high losses of fertilizers that also adversely impact on the environment. The presence of heavy elements in complex fertilizers in phosphate raw materials during superphosphate manufacturing also increases soil contamination. Consequently, the abovementioned problem becomes an urgent one in up-to-date agricultural production.

Decreasing the time of nutrient dissolution allows increasing the fertilizer use rate and preventing the accumulation of nitrates in the row layer. This fact can decrease the pollution of water systems due to the premature release of volatile nitrogen.

Thus, the purpose of the work is to create a reliable mathematical model of the process of washing out granular fertilizers from the soil, qualitatively and quantitatively reflecting the real physical process of mass transfer based on the parametric identification by the available experimental data. This model should allow determining 
the fertilizers concentration in soil over time, and estimating the time of the nitrogen release from soil.

\section{Literature Review}

The physical model of nutrients and heavy metals migration from encapsulated fertilizers can be represented by two stages [1]. The first is characterized by penetration of moisture from soil through the granule covering and consequent dissolution of the nitrogen nucleus, and diffusion of the supersaturated solution through the covering to the soil layer. In this process, the driving force is the difference between the nitrogen solution concentration inside the encapsulated fertilizer and in the soil. Phosphatecontaining coverings significantly reduce the concentration increase rate of nitrogen solution in the soil layer and limit the local zones with high nitrogen concentration.

The second stage begins after the complete dissolution of the granule's core and ends with the complete migration of nitrogen into the soil [2]. At the same time, highly-complicated processes of nitrogen transformation occur in the soil layer (i. e. dissolution, diffusion, migration, adsorption, and metabolism), which change the chemical composition of fertilizers and their distribution in the soil.

For the third stage, the simplified mathematical models are developed for describing the migration processes of nutrients in the soil layer [3-5]. These models allow predicting the kinetics of granule dissolution in the system "granule - moist porous medium". The calculation is based on the approximate determination of the diffusion coefficient of the dissolved substance inside the capsule and in the porous medium.

Currently, physical and mathematical models are known for describing the dissolution process of nitrogen-based components from the encapsulated granular fertilizers [6]. These models are based on studying the diffusion process through the spherical shell. However, the abovementioned research allows solving the first-order problem related to the definition of the mass transfer of nutrients from granule through the shell without the analytically-based methodology of estimating the time of the granule complete dissolution and kinetics of the nitrogen release from the capsule during the dissolution.

At the same time, in order to assess the ecological component of the process of washing out the components of agrochemicals in a row layer, a number of researchers apply separate mathematical models based on experimental investigations carried out in groundwater wells [7, 8]. The expediency of this method is based on the possibility of experimental fixation of water-soluble forms of substances in washing water. Particularly, in the research work [9], the internal spatial characteristics of the transport of substances in natural heterogeneous soils were studied for the further development of the corresponding mathematical model.

The research works $[10,11]$ is devoted to using the impact of microwave energy on the regeneration of the adsorbent. This approach can be applied to accelerate the process of mass transfer in fertilizers. Additionally, papers [12, 13] allow modeling the movement of granular material during its releasing from fertilizers.

A review of the technical approach based on the mathematical modeling and simulation of fertilizers release is presented in the works [14-16]. Additionally, the research paper [17] deals with modeling the diffusion process for a single granule for modeling of the controlled nutrient release from polymer-coated fertilizers, as well as the statistically based model for diffusion release is proposed in the research paper [18].

It should be noted that all the recent research does not allow developing the comprehensive mathematical model, the parameters of which can be determined by the results of numerical experimental data using the regression dependencies.

\section{Research methodology}

The analysis of experimental data obtained by the Research Institute of Mineral Fertilizers and Pigments of Sumy State University shows that the kinetics of washing out the nitrogen from the capsule can be described by the first-order differential equation with partial derivatives as the mass transfer equation describing the physical process of the change in the concentration of a substance over space and time [19]:

$$
\frac{\partial C(y, t)}{\partial t}=v \frac{\partial C(y, t)}{\partial y}+k\left[C_{*}-C(y, t)\right]
$$

where $C$ - concentration of nitrogen for a fertilizer dissolved in a granule, $\mathrm{mg} / \mathrm{kg} ; C_{*}-$ limiting concentration of nitrogen for a fertilizer dissolved in the granule, $\mathrm{mg} / \mathrm{kg}$; $t$ - time, weeks; $v$ - velocity of nitrogen release from the granule's cover, mm/week; $y$ - space coordinate, $\mathrm{mm}$; $k$-dissolution rate [20] evaluated experimentally, week ${ }^{-1}$.

Considering the potential ecological hazards from high fertilization rates, water-soluble nitrogen was chosen as the main component for ensuring the safety of fertilizers. The preliminary humidification of soil and the number of washing cycles were simulated by the natural weather conditions within the carried out agrochemical methods. In this 
case, the rate of dissolution of granules depends on the composition of phosphate-containing covering and its thickness.

Since the time of dissolution of the phosphate-containing covering is significantly higher in comparison on the time of nitrogen dissolution, when developing a mathematical model for the washing out nutrients from the encapsulated granule, the following assumption was considered [21-23]: coating material is difficult to dissolve and the physical properties remain unchanged during the period of dissolution of a nitrogen nucleus of fertilizer.

Consequently, the amount of the dissolved active substance in the soil is the main subject of the study. This allows simplifying the Eq. (1) to the form:

$\frac{d C(t)}{d t}=k\left[C_{*}-C(t)\right]$

Expressing the concentration $C=1-\Delta C$ by the parameter $\Delta C$ of nitrogen release from the soil during the fertilizers dissolution, the Eq. (2) after identical transformations takes the Eq. (3):

$\frac{d \Delta C}{d t}+k \Delta C=k \Delta C_{*}$,

where $\Delta C_{*}$ - limiting nitrogen loss from the soil in the process of fertilizers dissolution, $\mathrm{mg} / \mathrm{kg}$.

The general solution of the first-order linear non-homogeneous Eq. (3) for the initial condition $\Delta C(0)=\Delta C_{0}$ takes the Eq. (4) [24]:

$$
\Delta C(t)=\Delta C_{0} \exp (-k t)+\Delta C_{*}[1-\exp (-k t)]
$$

where $\Delta C_{0}-$ initial release of nitrogen from fertilizer, $\mathrm{mg} / \mathrm{kg}$.

The physical meaning of the limiting concentration is in the fact that the concentration $C \rightarrow C_{*}$ when $t \rightarrow \infty$. It should be noted that Eq. (3) and its solution Eq. (4) satisfy this requirement.

Under the simplifying assumption that the soil washing process out occurs relatively evenly during the fertilization period, a number of release cycles $N$ can be determined in terms of the corresponding frequency $f$, cycles / week, as $N=f \cdot t$. In this case, introducing the dimensionless empirical coefficient $\alpha=k / f$ allows rewriting the general solution Eq. (4) in Eq. (5):

$$
\Delta C(N)=\Delta C_{0} \exp (-\alpha N)+\Delta C_{*}[1-\exp (-\alpha N)] .
$$

Since the limiting concentration varies considerably for different granular fertilizers [25], there is a need for converting the mathematical model Eq. (6) to a unified one. Thus, the choice of the initial loss $\Delta C_{0}=0$ as an origin and introducing the dimensionless concentration:
$\Delta \bar{C}=\frac{\Delta C}{\Delta C_{*}} ; \quad 0 \leq \Delta \bar{C}<1$

allows obtaining the following unified model determined in terms of dimensionless parameters only:

$\Delta \bar{C}(N)=1-\exp (-\alpha N)$.

It should be noted that the parameters $\alpha$ is determined as a result of the regression analysis using the parameter identification of the developed mathematical model by the experimental results data $[25,26]$. For this purpose, procedures of quasi-linear parameter estimation and minimizing the error function determined by the least square method are implemented for the model Eq. (5):

$R(\alpha)=\sum_{i=1}^{n}\left[\alpha N_{i}-\frac{\ln \left(\Delta C_{*}-\Delta C_{i}\right)}{\Delta C_{*}-\Delta C_{0}}\right]^{2} \rightarrow \min$,

where $i$ - experiment number; $n$ - total number of the experimental data; $N_{i}, \Delta C_{i}$ - experimental values of the parameters for the physical process of mass transfer.

Equating to zero the derivative of the error function with respect to the unknown parameter:

$$
\frac{d R(\alpha)}{d \alpha}=2 \sum_{i=1}^{n}\left[\alpha N_{i}-\frac{\ln \left(\Delta C_{*}-\Delta C_{i}\right)}{\Delta C_{*}-\Delta C_{0}}\right] N_{i}=0
$$

allows obtaining a quasi-linear regression model for evaluating the dimensionless coefficient:

$$
\alpha=\frac{\sum_{i=1}^{n} N_{i} \frac{\ln \left(\Delta C_{*}-\Delta C_{i}\right)}{\Delta C_{*}-\Delta C_{0}}}{\sum_{i=1}^{n} N_{i}^{2}} .
$$

For the abovementioned unified model Eq. (8), the dependence Eq. (10) is significantly simplified:

$$
\alpha=\frac{\sum_{i=1}^{n} N_{i} \ln \left(1-\Delta \bar{C}_{i}\right)}{\sum_{i=1}^{n} N_{i}^{2}} .
$$

It should be noted that the parameter $\alpha$ can be determined directly by the experimental curve of soil washing as a tangent of the initial inclination angle of the curve "N- $\Delta C$ ". This fact is precisely justified by the following identity as a result of analytical analysis of the Eq. (7):

$$
\alpha=\lim _{N \rightarrow 0} \frac{d \Delta \bar{C}}{d N} .
$$

For each type of fertilizer, the coefficient $\alpha$ is determined separately. Its value depends on the degree of soil saturation with the active substance and the maximum level of 
washout. This fact is caused by different diffusion properties of the phosphate-containing membrane and its composition. Therefore, the dissolution rate for a granule's cover and the nitrogen release is significantly different [27]. Moreover, studies of encapsulated fertilizers [28, 29] show a decrease in the number of mobile forms of nitrogen.

The dependence Eq. (5) allows evaluating time $T$ of nitrogen release from the soil in terms of the permissible level of washout $[\Delta C], \mathrm{mg} / \mathrm{kg}$ :

$T=\frac{1}{k} \ln \frac{\Delta C_{*}-\Delta C_{0}}{\Delta C_{*}-[\Delta C]}$.

Particularly, for the values $\Delta C_{0}=0$ and $[\Delta C]=0.95 C_{*}$, it can be obtained $T_{0.95}=3 / k$. This dependence allows experimentally evaluating the dissolution rate $k=3 / T_{0.95}$, where $T_{0.95}$ is the time of $95 \%$ release of fertilizers (dissolution parameter $p=0.95$ ). For an arbitrary value of the parameter $p$, it can be written the dependence:

$k=\beta / T_{p}$,

where $\beta=-\ln (1-p)$ - dimensionless parameter presented in Fig. 1 and Table 1.

Particularly, for a half-dissolution period $T_{0.5}(p=0.5)$, the dissolution ratio $k=0.69 / t_{0.5}$.

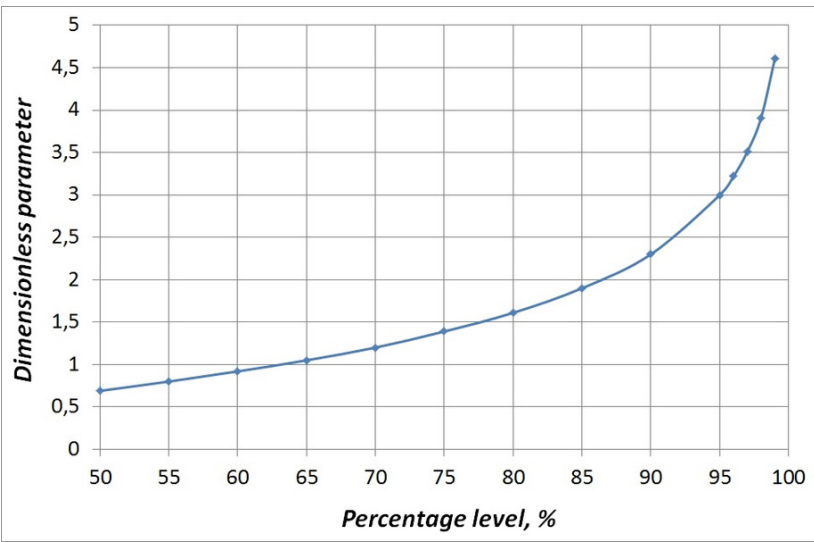

Fig. 1 The unified curve for evaluating the dissolution rate

Table 1 The parameter $\beta$ for evaluating the dissolution rate

\begin{tabular}{cccc}
\hline $\begin{array}{c}\text { Percentage } \\
\text { level, \% }\end{array}$ & Parameter $\beta$ & $\begin{array}{c}\text { Percentage } \\
\text { level, \% }\end{array}$ & Parameter $\beta$ \\
\hline 50 & 0.69 & 85 & 1.90 \\
55 & 0.80 & 90 & 2.30 \\
60 & 0.92 & 95 & 3.00 \\
65 & 1.05 & 96 & 3.22 \\
70 & 1.20 & 97 & 3.51 \\
75 & 1.39 & 98 & 3.91 \\
80 & 1.61 & 99 & 4.61 \\
\hline
\end{tabular}

\section{Results}

In the case of zero value of the initial nitrogen release, the results of theoretical calculations according to the mathematical model determined by Eq. (5) are presented in Fig. 2 for different values of the parameter $\alpha$ which corresponds to different types of fertilizers.

The features of the evaluated dependencies shown in Fig. 2 confirm the lowest rate of nitrogen washout. This fact allows resulting that the method of covering the granules by the combination of layers from superphosphate and calcium humate is the most efficient.

Fig. 3 presents the unified dimensionless dependencies for changes in the concentration of nitrogen washed out from the soil for different types of fertilizers. The presented curves are determined according to the mathematical model Eq. (7).

The rate of increase in the concentration of nitrogen in the wash solution corresponds to a higher value of the coefficient $\alpha$. This fact is characterized by an increase in the initial angle of the curves shown in Fig. 3.

When studying the process of dissolution of the nucleus of granules for encapsulated fertilizers and, consequently, of nitrogen losses from the soil, the approximating curves are built by the experimental points (Fig. 4) using the regression model based on the physical model Eq. (10) of mass transfer.

According to the comparison of data obtained using the mathematical modeling and experimental research, the carried out regression and correlation analysis proves

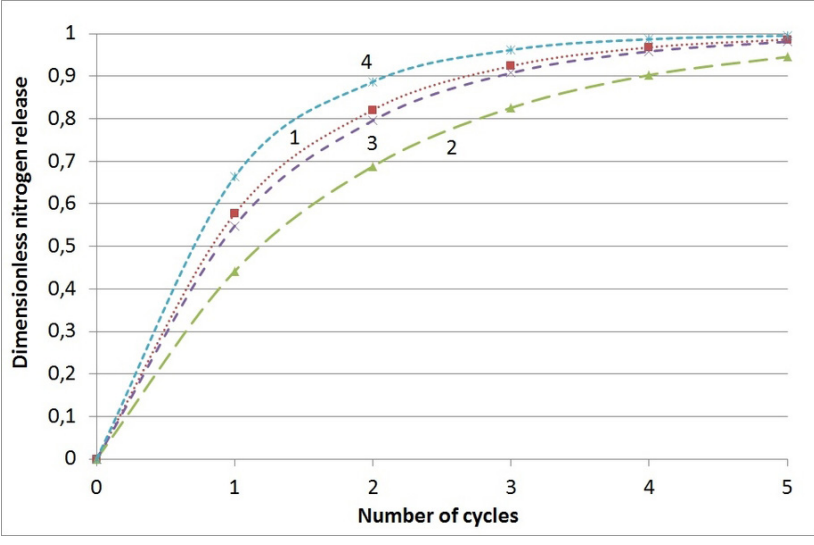

Fig. 2 Estimated curve of release of nitrogen fertilizers from a soil: 1 - carbamide; 2 - urea coated with superphosphate, plasticizer calcium humate; 3 - carbamide, coated with superphosphate and zeolite, plasticizer - calcium humate; 4 - carbamide, coated with superphosphate and zeolite, plasticizer $-50 \%$ solution of urea and calcium humate 


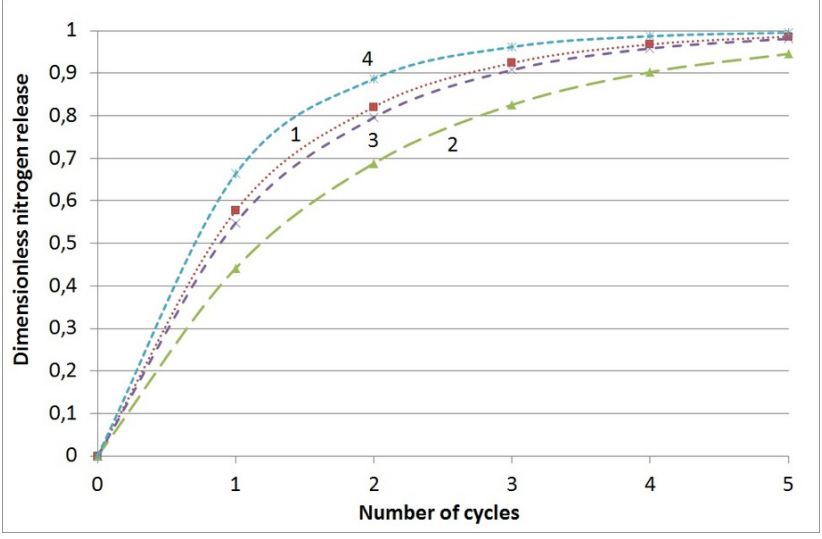

Fig. 3 A unified curve of release of nitrogen fertilizers from a soil: 1 - carbamide; 2 - urea coated with superphosphate, plasticizer calcium humate; 3 - carbamide, coated with superphosphate and zeolite, plasticizer - calcium humate; 4 - carbamide, coated with superphosphate and zeolite, plasticizer $-50 \%$ solution of urea and calcium humate

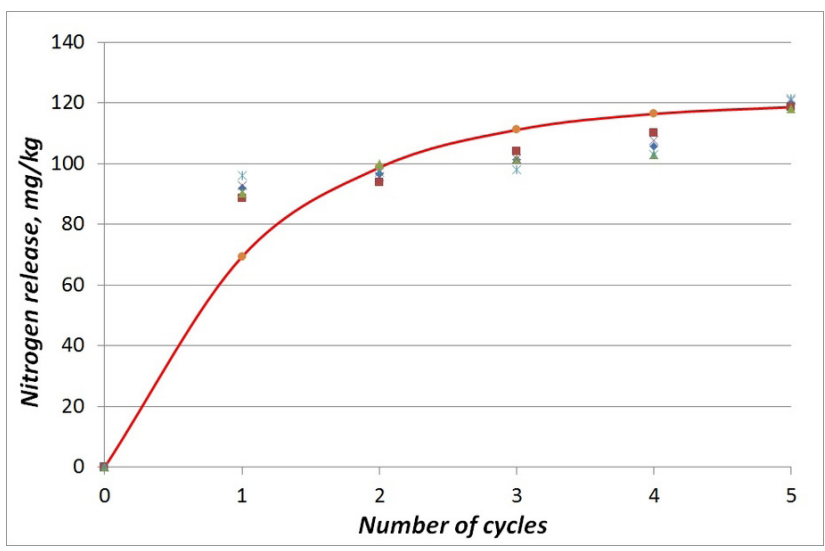

(a)

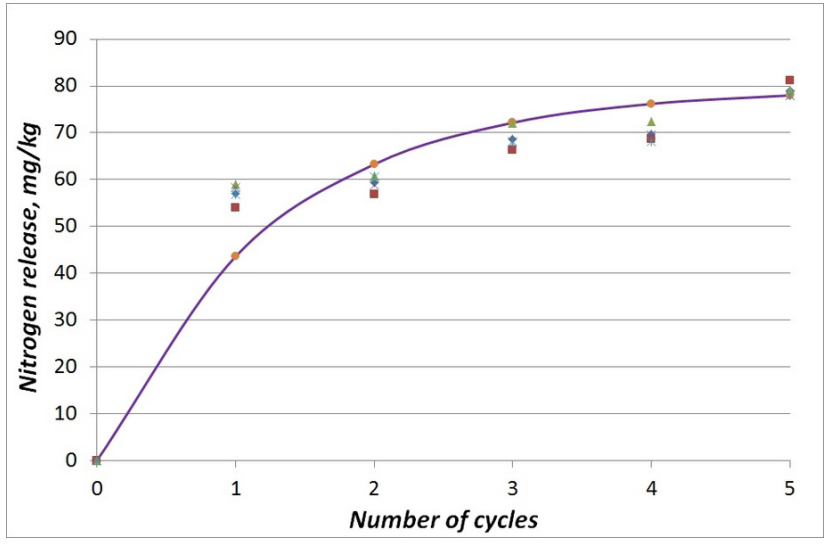

(c) a close correlation between the changes in nitrogen loss during the dissolution of the encapsulated fertilizer. As a result, correlation coefficients for the studied fertilizers are equal to $0.964,0.996,0.971$, and 0.981 for the cases presented in Fig. 4.

According to the calculated mathematical model, the use of fertilizers with the test coatings reduces the loss of active ingredient due to leaching during natural precipitation and prolongs the use of nutrients by plants.

When using urea, the amount of washed nitrogen will be at least $118.7 \mathrm{mg} / \mathrm{kg}$; carbamide coated with superphosphate with a plasticizer with calcium humate - not less than $33.7 \mathrm{mg} / \mathrm{kg}$; urea coated with superphosphate and zeolite with a plasticizer with calcium humate - not less than $78 \mathrm{mg} / \mathrm{kg}$; carbamide, coated with superphosphate and zeolite, plasticizer solution of urea and calcium humate - not less than $80.8 \mathrm{mg} / \mathrm{kg}$. Thus, it is possible

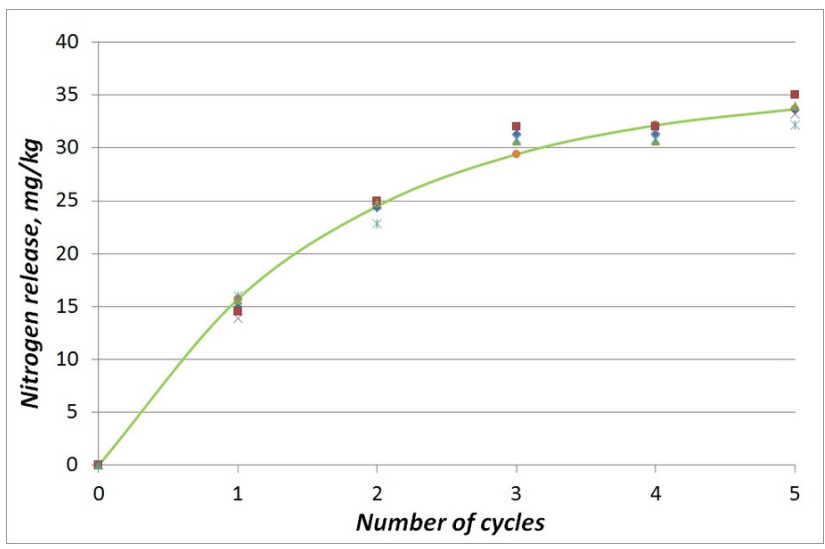

(b)

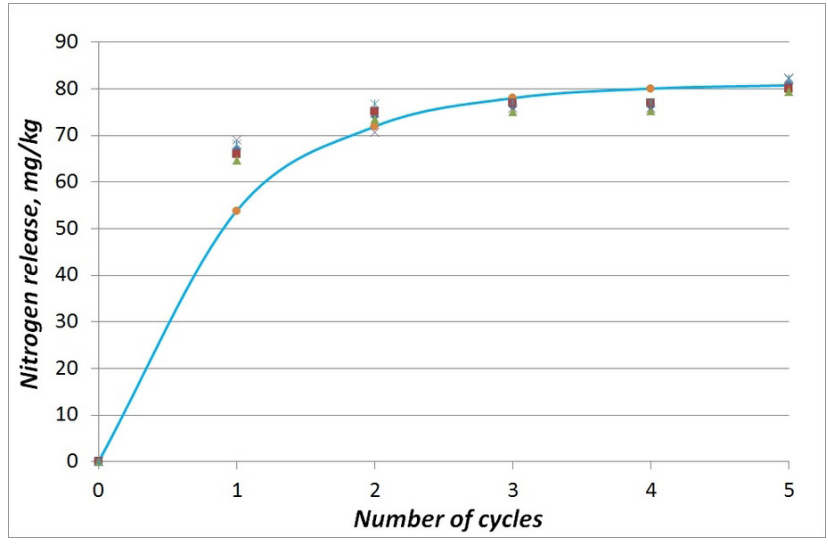

(d)

Fig. 4 Release of nitrogen fertilizers from a soil: (a) carbamide; (b) urea coated with superphosphate, plasticizer - calcium humate; (c) carbamide, coated with superphosphate and zeolite, plasticizer - calcium humate; (d) carbamide, coated with superphosphate and zeolite, plasticizer $-50 \%$ solution of urea and calcium humate 
to reduce the loss of nitrogen from fertilizer in 1.5-3.5 times. This will allow, respectively, to reduce the number of applied fertilizers and, as a result, the load on the soil and the aquatic environment and to minimize the oversaturation of plants with nitrogen.

\section{Conclusions}

An analysis of the dissolution characteristics of the capsular granule nucleus has been performed to reduce the amount of water-soluble nitrogen and the rate of its accumulation in the soil layer. The problem is formulated considering environmental constraints and solved using the proposed reliable mathematical model. The possibility of reducing the ecological load on the environment by applying nitrogen fertilizers having different diffusion properties of the phosphate-containing membrane and composition is substantiated and solved by investigating the kinetics of the washing out of water-soluble nitrogen fertilizers. As a result, the operating parameters of the process are determined. The results of the developed mathematical model allow calculating the change in the concentration of nitrogen washed out of different types of fertilizers over time. The carried out simulations allow evaluating the efficiency of fertilizers of prolonged action and environmental impact on the environment.

\section{References}

[1] Zhao, L., Zhang, Z., Tang, Z., Feng, S. "Migration characteristics of mineral nitrogen with surface runoff in dry farmland soil", Acta Scientiae Circumstantiae, 30(10), pp. 1977-1984, 2010.

[2] Dube, A., Zbytniewski, R., Kowalkowski, T., Cukrowska, E., Buszewski, B. "Adsorption and Migration of Heavy Metals in Soil", Polish Journal of Environmental Studies, 10(1), pp. 1-10, 2001. [online] Available at: http://www.pjoes.com/ pdf-87344-21203?filename=Adsorption $\% 20$ and\%20migration.pdf [Accessed: 15 January 2019]

[3] Shandyba, A. B. "Geotechnical and environmental assessment of contaminated sites under migration of polluting components", In: $4^{\text {th }}$ International Symposium on Geotechnics Related to the Environment, GREEN 4, Wolverhampton, UK, 2004, pp. 173-177. https://doi.org/10.1201/9780367803346

[4] Nagursky, O., Gumnitsky, Y. "Theoretical Model of Compounds Release from Capsulated Particles and Its Experimental Check", Chemistry and Chemical Technology, 6(1), pp. 101-103, 2012. [online] Available at: http://science2016.lp.edu.ua/sites/default/ files/Full_text_of_\%20papers/full_text_442.pdf [Accessed: 15 January 2019]

[5] Gumnitskii, Y. M., Lyuta, O. V. "Molecular-diffusion mass transfer of substance in soil medium", Theoretical Foundations of Chemical Engineering, 48(4), pp. 414-419, 2014.

https://doi.org/10.1134/S0040579514040174
The scientific and practical significance of the obtained results is in developed regression dependencies for determining the dissolution rate and time to wash out fertilizers by the results of experimental research. The reliability of the proposed mathematical model is justified by the relatively high correlation coefficients in a range of 0.96-0.99 for the experimentally studied fertilizers.

\section{Acknowledgments}

The theoretical part of the research was provided by the project "Development and Implementation of Energy Efficient Modular Separation Devices for Oil and Gas Purification Equipment" (state reg. no. 0117U003931) ordered by the Ministry of Education and Science of Ukraine.

The numerical simulation results were obtained at the Faculty of Manufacturing Technologies with a seat in Presov of Technical University of Kosice (Presov, Slovak Republic) within the research project "Identification of Parameters for Technological Equipment using Artificial Neural Networks" funded by the National Scholarship Programme of the Slovak Republic.

The experimental results were obtained by the Research Institute of Mineral Fertilizers and Pigments of Sumy State University (Ukraine).

[6] Basu, S. K., Kumar, N. "Mathematical model and computer simulation for release of nutrients from coated fertilizer granules", Mathematics and Computers in Simulation, 79(3), pp. 634-646, 2008.

https://doi.org/10.1016/j.matcom.2008.04.012

[7] Marinov, I., Marinov, A. M. "A Coupled Mathematical Model to Predict the Influence of Nitrogen Fertilization on Crop, Soil and Groundwater Quality", Water Resources Management, 28(15), pp. 5231-5246, 2014.

https://doi.org/10.1007/s11269-014-0664-5

[8] Filipović, V., Kodešová, R., Petošić, D. "Experimental and mathematical modeling of water regime and nitrate dynamics on zero tension plate lysimeters in soil influenced by high groundwater table", Nutrient Cycling in Agroecosystems, 95(1), pp. 23-42, 2013. https://doi.org/10.1007/s10705-012-9546-5

[9] Sheng, F., Wang, K., Zhang, R., Liu, H. "Modeling the heterogeneous soil water flow and solute transport by two-region-two-stage model", Journal of Hydraulic Engineering, 46(4), pp. 433-442, 2015. (in Chinese) https://doi.org/10.13243/j.cnki.slxb.2015.04.007

[10] Dobrotvorskiy, S., Aleksenko, B., Dobrovolska, L., Basova, Y. "Effect of the Application of Microwave Energy on the Regeneration of the Adsorbent", Acta Polytechnica, 58(4), pp. 217-225, 2018. https://doi.org/10.14311/AP.2018.58.0217 
[11] Dobrotvorskiy, S., Dobrovolska, L., Aleksenko, B., Basova, Y. "The Use of Waveguides with Internal Dissectors in the Process of Regeneration of Industrial Adsorbents by Means of the Energy of Ultrahigh-Frequency Radiation", In: Ivanov, V., Rong, Y., Trojanowska, J., Venus, J., Liaposhchenko, O., Zajac, J., Pavlenko, I., Edl, M., Perakovic, D. (eds.) Advances in Design, Simulation and Manufacturing, Lecture Notes in Mechanical Engineering, Springer, Cham, Switzerland, pp. 433-442, 2019. https://doi.org/10.1007/978-3-319-93587-4_45

[12] Hyvlud, A., Sabadash, V., Gumnitsky, J., Ripak, N. "Statics and Kinetics of Albumin Adsorption by Natural Zeolite", Chemistry and Chemical Technology, 13(1), pp. 95-100, 2019. https://doi.org/10.23939/chcht13.01.095

[13] Bahrami, A., Hosseinzadeh, S., Ghasemiasl, R., Radmanesh, M. "Solution of Non-Fourier Temperature Field in a Hollow Sphere under Harmonic Boundary Condition", Applied Mechanics and Materials, 772, pp. 197-203, 2015.

https://doi.org/10.4028/www.scientific.net/AMM.772.197

[14] Irfan, S. A., Razali, R., KuShaari, K., Mansor, N., Azeem, B., Ford Versypt, A. N. "A review of mathematical modeling and simulation of controlled-release fertilizers", Journal of Controlled Release, 271, pp. 45-54, 2018.

https://doi.org/10.1016/j.jconrel.2017.12.017

[15] Du, C., Zhou, J., Shaviv, A., Wang, H. "Mathematical Model for Potassium Release from Polymer-coated Fertiliser", Biosystems Engineering, 88(3), pp. 395-400, 2004. https://doi.org/10.1016/j.biosystemseng.2004.03.004

[16] Lan, R., Liu, Y., Wang, G., Wang, T., Kan, C., Jin, Y. "Experimental modeling of polymer latex spray coating for producing controlled-release urea", Particuology, 9(5), pp. 510-516, 2011. https://doi.org/10.1016/j.partic.2011.01.004

[17] Shaviv, A., Raban, S., Zaidel, E. "Modeling Controlled Nutrient Release from Polymer Coated Fertilizers: Diffusion Release from Single Granules", Environmental Science and Technology, 37(10), pp. 2251-2256, 2003. https://doi.org/10.1021/es011462v

[18] Shaviv, A., Raban, S., Zaidel, E. "Modeling Controlled Nutrient Release from a Population of Polymer Coated Fertilizers: Statistically Based Model for Diffusion Release", Environmental Science and Technology, 37(10), pp. 2257-2261, 2003. https://doi.org/10.1021/es0205277

[19] Pavlenko, I. V., Yukhymenko, M. P., Lytvynenko, A. V., Bocko, J. "Solving the Nonstationary Problem of the Disperse Phase Concentration during the Pneumoclassification Process of Mechanical Mixtures", Journal of Engineering Sciences, 6(1), pp. F1-F5, 2019. https://doi.org/10.21272/jes.2019.6(1).f1

[20] Sklabinskyi, V., Liaposhchenko, O., Pavlenko, I., Lytvynenko, O., Demianenko, M. "Modelling of Liquid's Distribution and Migration in the Fibrous Filter Layer in the Process of Inertial-Filtering
Separation", In: Ivanov, V., Rong, Y., Trojanowska, J., Venus, J., Liaposhchenko, O., Zajac, J., Pavlenko, I., Edl, M., Perakovic, D. (eds.) Advances in Design, Simulation and Manufacturing, Lecture Notes in Mechanical Engineering, Springer, Cham, Switzerland, pp. 489-497, 2019.

https://doi.org/10.1007/978-3-319-93587-4_51

[21] Nagursky, O., Gumnitsky, Y. "Mass Exchange of Dispersed Materials Encapsulating in Quasi-Liquefaction State", Chemistry and Chemical Technology, 9(3), pp. 333-336, 2015. https://doi.org/10.23939/chcht09.03.333

[22] Nagursky, O., Gumnitsky, Y., Vaschuk, V. "Unsteady Heat Transfer during Encapsulation of Dispersed Materials in Quasi-liquefied State", Chemistry and Chemical Technology, 9(4), pp. 497-501, 2015. https://doi.org/10.23939/chcht09.04.497

[23] Yuan, G. "An organoclay formula for the slow release of soluble compounds", Applied Clay Science, 100, pp. 84-87, 2014. https://doi.org/10.1016/j.clay.2014.04.005

[24] Ostroha, R., Yukhymenko, M., Yakushko, S., Artyukhov, A. "Investigation of the Kinetic Laws Affecting the Organic Suspension Granulation in the Fluidized Bed", Eastern-European Journal of Enterprise Technologies, 4(1), pp. 4-10, 2017. https://doi.org/10.15587/1729-4061.2017.107169

[25] Tzika, M., Alexandridou, S., Kiparissides, C. "Evaluation of the morphological and release characteristics of coated fertilizer granules produced in a Wurster fluidized bed", Powder Technology, 132(1), pp. 16-24, 2003. https://doi.org/10.1016/S0032-5910(02)00345-5

[26] Yousef Nezhad, M. E., Hoseinzadeh, S. "Mathematical modelling and simulation of a solar water heater for an aviculture unit using MATLAB / SIMULINK", Journal of Renewable and Sustainable Energy, 9(6), article ID: 063702, 2017. https://doi.org/10.1063/1.5010828

[27] Zheng, W., Zhang, M., Liu, Z., Zhou, H., Lu, H., Zhang, W., Yang, Y., Li, C., Chen, B. "Combining controlled-release urea and normal urea to improve the nitrogen use efficiency and yield under wheat-maize double cropping system", Field Crops Research, 197, pp. 52-62, 2016.

https://doi.org/10.1016/j.fcr.2016.08.004

[28] Sun, Y.-G., Sun, Z. "Characteristics of biodegradable puffed slow-release fertilizers", Journal of Clinical Rehabilitative Tissue Engineering Research, 12(10), pp. 1991-1994, 2008.

[29] Ostroha, R., Yukhymenko, M., Lytvynenko, A., Bocko, J., Pavlenko, I. "Granulation Process of the Organic Suspension: Fluidized Bed Temperature Influence on the Kinetics of the Granule Formation", In: Ivanov, V., Rong, Y., Trojanowska, J., Venus, J., Liaposhchenko, O., Zajac, J., Pavlenko, I., Edl, M., Perakovic, D. (eds.) Advances in Design, Simulation and Manufacturing, Lecture Notes in Mechanical Engineering, Springer, Cham, Switzerland, pp. 463-471, 2019.

https://doi.org/10.1007/978-3-319-93587-4_48 